\title{
AATF is Overexpressed in Human Bladder Cancer and Regulates Chemo-Sensitivity Through Survivin
}

\author{
Shutao Tan' \\ Lin $\mathrm{Fu}^{2}$ \\ Qianze Dong ${ }^{2}$ \\ 'Department of Urology, Shengjing \\ Hospital of China Medical University, \\ Shenyang, People's Republic of China; \\ ${ }^{2}$ Department of Pathology, College of \\ Basic Medical Science, China Medical \\ University and Department of Pathology, \\ The First Affiliated Hospital of China \\ Medical University, Shenyang, People's \\ Republic of China
}

Objective: Dysregulation of apoptosis antagonizing transcription factor (AATF) has been reported to be closely associated with human cancers. However, its involvement in human bladder cancer $(\mathrm{BC})$ remains unexplored. This study aimed to investigate the clinical significance and biological roles of AATF in human bladder cancers.

Methods: AATF protein expression was examined in 107 cases of bladder cancer tissues using immunohistochemistry. AATF plasmid transfection and small interfering RNA (siRNA) knockdown were performed in T24 and 5637 cell lines. CCK-8, colony formation, annexin V/PI, JC-1 staining, and Western blotting were carried out to investigate the biological roles and underlying mechanisms of AATF in bladder cancer cells.

Results: Our results showed that AATF expression was upregulated in human bladder cancer specimens and correlated with $\mathrm{T}$ stage. Analysis of the Oncomine database showed elevation of AATF mRNA in BC tissues. The Cancer Genome Atlas (TCGA) data suggested that high AATF expression correlated with poor patient survival. Western blotting showed that AATF protein expression was higher in BC cell lines compared to normal bladder transitional epithelial cell line SV-HUC-1. CCK-8 and colony assays showed that ectopic AATF expression upregulated cell growth rate and colony numbers. CCK-8, annexin V/ propidium iodide (PI), JC-1 assays and Western blotting showed that AATF overexpression decreased cisplatin sensitivity, downregulated cisplatin-induced apoptosis and upregulated mitochondrial membrane potential, with decreased cytochrome $\mathrm{c}$ and cleaved-PARP expression. AATF siRNA knockdown showed the opposite effects. Mechanistically, AATF overexpression upregulated cyclin E and Survivin at both mRNA and protein levels. The decreased cisplatin sensitivity/apoptosis induced by ectopic AATF were reversed after treatment with Survivin inhibitor YM155.

Conclusion: Our results showed that AATF was overexpressed in human bladder cancers and promoted malignant behavior by regulating cyclin E and Survivin, indicating AATF could serve as a malignant biomarker and potential therapeutic target in BC.

Keywords: AATF, apoptosis, bladder cancer, survivin

\section{Introduction}

Bladder cancer (BC) is the most common urinary tract malignancy and the leading causes of cancer-related death worldwide. ${ }^{1,2}$ Despite introduction of more effective therapeutic regimens including surgery and chemotherapy, the prognosis of patients with advanced stage $\mathrm{BC}$ remains poor. ${ }^{3}$ One of the most important causes of BCrelated death was development of chemoresistance, which lead to the failure of chemotherapy. ${ }^{4}$ Thus, identifying the mechanisms underlying of chemoresistance development and seeking more effective targets involved in chemoresistance is of great importance. ${ }^{5}$
Correspondence: Qianze Dong Department of Pathology, College of Basic Medical Science, China Medical

University and Department of Pathology,

The First Affiliated Hospital of China

Medical University, Shenyang, People's

Republic of China

Email dongqianze@hotmail.com 
The transcriptional cofactor Apoptosis Antagonizing Transcription Factor (AATF) is currently considered to play an important role in the DNA damage response (DDR), which maintains genome integrity. ${ }^{6}$ AATF was originally reported as a transcriptional co-factor which was involved gene regulation by connecting transcription factor to the general transcriptional machinery. ${ }^{6}$ AATF has been shown to interact with transcription factors such as p65 and Signal transducer and activator of transcription 3 (STAT3). ${ }^{7,8}$ Recent studies found AATF expression was dysregulated in various cancers. ${ }^{9}$ AATF could affect the p53 activity and its target specificity. ${ }^{10}$ AATF was reported to be amplified in neuroblastoma, the level of which was associated with poor patient prognosis. ${ }^{11}$ AATF could promote multiple myeloma proliferation by affecting chromatin structure. ${ }^{12}$ However, the biological roles of AATF and its expression pattern in bladder cancer have not been explored. In addition, its potential biological mechanisms in cancer cells need to be elucidated. In this study, we examined the clinical significance of AATF in BC tissues and explored its biological roles in $\mathrm{BC}$ cell lines.

\section{Materials and Methods}

\section{Specimens}

This study was approved by the ethics review board of China Medical University (Approval number: 2020PS157K). Participants provided written informed consent and the study was performed according to the principles in the Declaration of Helsinki. The paraffinembedded bladder cancer specimens were obtained from the Pathology archives of the First Affiliated Hospital of China Medical University, which contain specimens no longer required to be maintained.

\section{Immunohistochemistry}

Sections (4- $\mu \mathrm{m}$ thick) were firstly treated in xylene to deparaffinize, followed by graded ethanol to rehydrate. Endogenous peroxidase was blocked by $\mathrm{H}_{2} \mathrm{O}_{2}$ solution. Heat antigen retrieval was carried out using citrate buffer $(\mathrm{pH}=6.0)$. After incubation with primary AATF antibody (1:200, Proteintech, Rosemont, IL, USA), the sections were incubated with ElivisionPlus HRP secondary antibody Polymer (Maixin, Fuzhou, China). Then slides were stained by DABplus kit (Maixin, Fuzhou, China).

Immunostaining was evaluated by two pathologists from the pathology department in the First Affiliated Hospital of China Medical University (Q.D. and L.F.). Staining for each of the proteins was scored using the methods of modified immunoreactive score (IRS). ${ }^{13,14}$ Nuclear staining was regarded as positive staining. The intensity of staining was scored as 0 negative/weak; 1 moderate; 2 strong. The percentage of positive expression was categorized as: 1 $(<25 \%) ; 2$ (25-50\%); 3 (50-75\%); 4 (75-100\%). The final scores were obtained by multiplying the intensity scores with the percentage scores. Samples with scores $<4$ were considered as low expression. Samples with scores between 4 and 8 were considered as high expression.

\section{Cell Culture and Transfection}

SV-HUC-1, T24, 5637, J82 cell lines were obtained from Shanghai cell bank of Chinese Academy of Sciences (Shanghai, China). Cell lines were maintained in RPMI1640 with $10 \%$ fetal bovine serum (FBS) in an incubator with $5 \% \mathrm{CO}_{2}$. AATF plasmid and control vector were purchased from Origene (Origene, Montgomery, Maryland, USA). Lipofectamine 3000 (Invitrogen, Carlsbad, CA, USA) was used for plasmid transfection. AATF small interfering siRNA (GGACUUGGAUGAA GAAAUC) was purchased from Dharmacon (Horizon, Lafayette, CO, USA). Dharmafect1 reagent (Horizon, Lafayette, CO, USA) was used for siRNA transfection. Cisplatin and YM155 were obtained from Selleck Chemicals (Houston, TX, USA).

\section{Quantitative RT-PCR}

Total RNA was extracted using TRIzol reagent. After quantification, RNA was reverse transcribed using PrimeScript RT Master Mix Kit (Takara, Dalian, Liaoning, China). SYBR select master Mix Kit (Thermo, Waltham, MA, USA) was used for qPCR, and the reaction was conducted as follows: $96^{\circ} \mathrm{C}$ for 3 minutes, 40 cycles of $96^{\circ} \mathrm{C}$ for 20 seconds and $62^{\circ} \mathrm{C}$ for 30 seconds. Relative expression of AATF was normalized to GAPDH and calculated according to $2^{-\Delta \Delta c t}$. This experiment was repeated in triplicate. The primers were designed using Oligo primer analysis software and synthesized by Sangon Biotechnology (Shanghai, China).

The sequences of primers were listed as follows: AATF for 5'-CAGTCTACAGGAACCGCACAC-3', AATF rev 5'-GTTCAAAGGCACCAAAACCCT-3'; cyclin E1 for 5'AGCCAGCCTTGGGACAATAAT-3', cyclin E1 rev 5'-G AGCCTCTGGATGGTGCAAT-3'; Survivin for 5'- CCCA CTGAGAACGAGCCAGA -3', Survivin rev 5'- CCAGC CTTCCAGCTCCTTG -3'; GADPH for 5'-GAAATCC 


\section{CATCACCATCTTCCAG-3', GADPH rev 5'-GAGCCC CAGCCTTCTCCAT-3'.}

\section{Western Blotting}

Total protein lysate was harvested using Radioimmunoprecipitation assay buffer (RIPA buffer) with protease inhibitors. Forty-microgram proteins were separated by SDS-PAGE and transferred to polyvinylidene fluoride (PVDF) membranes (Millipore, Burlington, MA, USA). The PVDF membranes were incubated with primary antibodies AATF (1:1000, Proteintech), cytochrome c, cleaved-PARP, cyclin E, Survivin (1:1000, Cell Signal Technology, Danvers, MA, USA), Actin (1:2000, Santa Cruz, Dallas, Texas, USA). The PVDF membranes were washed with Tris-buffered saline with $0.1 \%$ Tween 20 Detergent (TBST) and incubated with HRP-linked Anti-rabbit IgG or HRP-linked Anti-mouse IgG (1:2000, Cell Signal Technology). Visualization was performed using Enhanced chemiluminescence (ECL, Thermo) and DNR Bio-Imaging Systems (DNR, Neve Yamin, Israel).

\section{CCK-8 and Colony Formation Assays}

For CCK-8 assay, cells were plated in 96-well plates in medium containing $10 \%$ FBS at approximately 3000 cells per well $24 \mathrm{~h}$ after transfection. Ten microliters of CCK-8 reagent (Cell Counting Kit-8; Dojindo, Kumamoto, Japan) was added into each well. After incubation for 2 hours, absorbance was examined using a microplate reader (wavelength: $450 \mathrm{~nm}$ ). For colony formation capacity, cells were seeded into 6 -cm plates (1000 cells per plate) and cultured for about 2 weeks. The cells were then stained using Giemsa and counted under a microscope.

\section{Annexin V/PI Apoptosis Staining}

For apoptosis assay, cells were stained with $5 \mathrm{mg} / \mathrm{mL}$ propidium iodide together with Annexin V/FITC (BD bioscience, Franklin Lakes, NJ, USA). The cell solution was incubated in the dark for 15 minutes, and flow cytometry (ACEA Novocyte, Santa Clara, CA, USA) was used to detect the fluorescence.

\section{Cell Cycle Analysis}

A $0.5 \%$ trypsin solution was used to dissociate cells from culture plates. Binding buffer $(250 \mu \mathrm{L})$ was used for the resuspension of cells. For the cell cycle assay, the cells were stained in $5 \mathrm{mg} / \mathrm{mL}$ propidium iodide and analyzed by flow cytometry.

\section{Mitochondrial Membrane Potential}

Mitochondrial membrane potential was measured using JC-1 staining followed by flow cytometry. Cells were treated with JC-1 staining solution at a concentration of $5 \mu \mathrm{M}$ (Abcam, Waltham, MA, USA) for 30 minutes in the incubator. Then stained cells were washed twice and examined using ACEA flow cytometer.

\section{Luciferase Reporter Assay}

To construct pGL3-survivin-promoter-Luc plasmid, -1828 to +81 fragment of the human survivin promoter was amplified and inserted into the pGL3 plasmid. BC cells were transfected with pGL3-survivin-promoter-Luc DNA together with AATF plasmid or siRNA. Forty-eight hours after transfection, luciferase activity was assessed using the Promega luciferase assay Kit (Madison, WI, USA).

\section{Statistical Analysis}

Statistical analysis was carried out using the software package SPSS, version 16.0 (SPSS, Chicago, IL, USA). A $\chi 2$ test was used to analyze the possible associations between AATF expression and clinicopathological parameters. Overall survival was visualized using KaplanMeier and compared by Log rank test. Differences in gene expression, proliferation, apoptosis rate were assessed using the Student's $t$-test. $\mathrm{P}<0.05$ was considered to indicate statistical significance.

\section{Results}

\section{AATF Was Overexpressed in Human Bladder Cancers}

To investigate the clinical significance of AATF in human BC, AATF protein levels were examined using immunohistochemistry in 107 cases of $\mathrm{BC}$ tissues and 12 cases of normal bladder tissues. We found weak AATF immunostaining in normal urothelial tissues (Figure 1A). Increased nuclear AATF staining was found in 52 of 107 cases of bladder cancer (Figure 1B-D). The magnified image in Figure 1C shows nuclear staining, which was indicated using arrows in Supplementary Figure 1A. Negative controls using IgG showed no nuclear staining. The corresponding negative controls for Figure $1 \mathrm{C}$ and D are shown in Supplementary Figure 1B and $\underline{\mathrm{C}}$. As listed in Table 1, statistical significance was found between high AATF expression and $\mathrm{T}$ stage (Ta-T1 vs T2-T4, $\mathrm{p}=0.013$ ). The associations between AATF levels and other parameters did not reach statistical significance. 
A

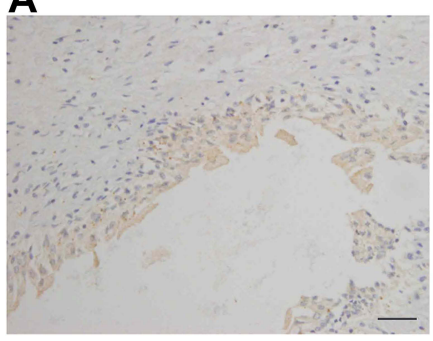

C

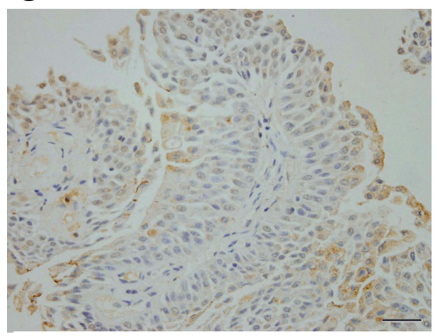

E

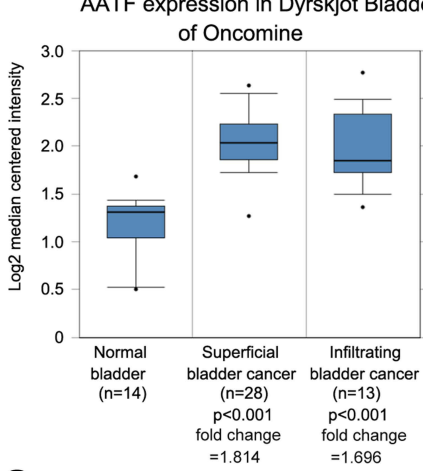

G

AATF expression in Sanchez-Carbayo Bladder 2

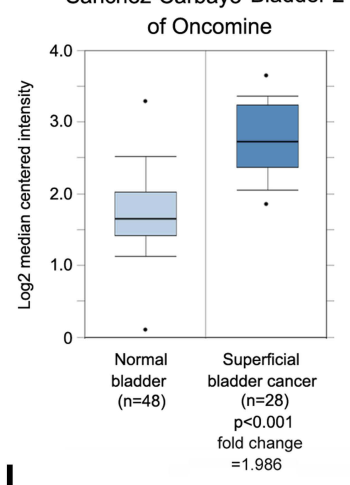

I
B

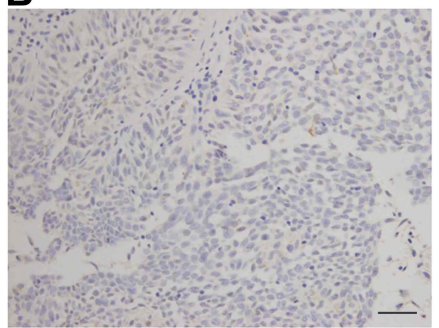

D

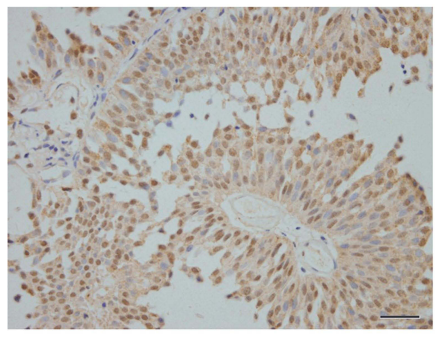

$\mathbf{F}$

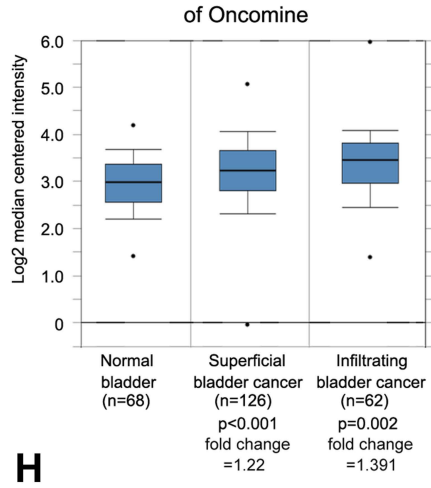

TCGA survival function

Log Rank test $p=0.0024$
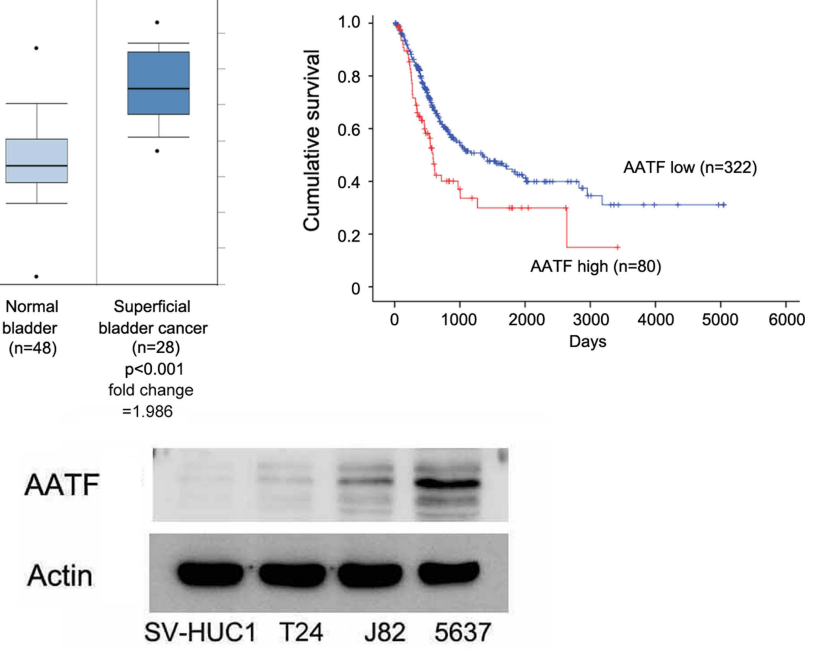

Figure I Expression of AATF in bladder cancers. (A) Weak AATF staining in normal bladder uroepithelial tissue. (B) Negative AATF staining in a bladder cancer. (C) Moderate AATF staining in a case of bladder cancer. (D) Strong cytoplasmic and nuclear AATF staining in a case of bladder cancer. (E) Analysis of Dyrskjot bladder 3 Oncomine dataset suggested that AATF mRNA was increased in bladder cancers compared with normal bladder tissues. (F) Analysis of Lee bladder dataset suggested that AATF mRNA was higher in bladder cancer tissues compared with normal bladder tissues. (G) Analysis of Sanchez-Carbayo bladder 2 suggested that AATF mRNA was higher in superficial bladder cancers compared with normal bladder. (H) Analysis of TCGA data using Kaplan-Meier curves showed that high AATF levels was associated with poor survival ( $P=0.0024$, Log rank test) in bladder cancer patients. (I) Western blotting showed that AATF protein was low in normal uroepithelial cell line SV-HUC-I and higher in BC cell lines J82 and 5637. (Magnification: 400x; bars indicates 50 $\mathrm{mm}$ ). 
Table I Distribution of AATF in Bladder Cancer According to Clinicopathological Characteristics

\begin{tabular}{|l|c|c|c|c|}
\hline Characteristics & Number of Patients & AATF Low Expression & AATF High Expression & P \\
\hline Age & & & & \\
$<60$ & 45 & 21 & 24 & 0.4038 \\
$\geq 60$ & 62 & 34 & 28 & \\
\hline Gender & 29 & 12 & 17 & 0.2059 \\
Female & 78 & 43 & 35 & \\
Male & & & & 0.0130 \\
\hline T stage & 46 & 30 & 36 & \\
Ta-TI & 61 & 25 & & 0.4730 \\
T2-T4 & & 26 & 21 & \\
\hline Tumor grade & 47 & 29 & 31 & \\
G1 & 60 & & & \\
G2 and G3 & &
\end{tabular}

We also analyzed data from the Oncomine database. The Dyrskjot bladder 3 dataset revealed that AATF mRNA was significantly elevated in $\mathrm{BC}$ compared with normal bladder (Figure 1E). Lee bladder and Sanchez-Carbayo bladder 2 of Oncomine also showed the similar results (Figure 1F and G). Next, we analyzed of the RNA-seq data of bladder cancers from the Tumor Genome Atlas (TCGA). The result showed that higher AATF levels significantly correlated with poor patient survival $(p=0.0024)$ (Figure $1 \mathrm{H}$ ). Together, our data indicated AATF expression was elevated in human $\mathrm{BC}$ and correlated with malignant features.

AATF protein expression was examined in 3 bladder cancer cell lines including T24, 5637, J82 and the immortalized human uroepithelial cell line SV-HUC-1. Western blotting showed that endogenous AATF protein was relatively higher in bladder cancer cell lines (especially in 5637 cell line) compared with normal SV-HUC-1 cell line (Figure 1I).

\section{AATF Promoted BC Cell Proliferation}

We selected T24 cell line with low endogenous AATF for plasmid transfection and 5637 cell line for AATF siRNA transfection. The efficiency of plasmid and siRNA transfection was validated using RT-qPCR and Western blotting (Figure 2A).

We then performed CCK8 and colony formation assays. CCK-8 assays showed that AATF depletion downregulated the growth rate while AATF plasmid transfection increased the cellular growth rate (Figure 2B). Colony formation assay demonstrated that AATF knockdown decreased colony formation ability while AATF transfection upregulated colony formation ability (Figure 2C). Cell cycle analysis was also performed. As shown in Figure 3A, AATF overexpression upregulated S percentage in T24 cell line, while AATF siRNA decreased $\mathrm{S}$ percentage in 5637 cells. These results indicated that AATF promoted cell proliferation possibly through cell cycle control. We also overexpressed AATF in 5637 cell line and silenced AATF in T24 cell line (Supplementary Figure 2A). AATF silencing reduced T24 cell growth (Supplementary Figure 2B) and colony formation ability (Supplementary Figure 2C). Overexpression of AATF increased 5637 cell growth (Supplementary Figure 2B) and colony formation ability (Supplementary Figure 2C), but the effect was not as strong as that in T24 cells, probably due to the high endogenous AATF in 5637 cells.

\section{AATF Regulates Cisplatin Sensitivity and Apoptosis in Bladder Cancer Cells}

The involvement of AATF in apoptosis and drug sensitivity of bladder cancer cells have not been fully explored. Next, we investigated the involvement of AATF in the regulation of cisplatin sensitivity of $\mathrm{BC}$ cells.

Using CCK8 cell viability assay, we checked the change of inhibition rate of different concentration of cisplatin (24h treatment) in AATF overexpressed and depleted cells. T24 cells with ectopic AATF expression showed decreased sensitivity and increased IC50 compared with control (IC50 con vs AATF: $12.4 \mu \mathrm{M}$ vs $14.5 \mu \mathrm{M}, \mathrm{p}<0.05$ ) (Figure 3B). 5637 cells with AATF depletion showed increased cisplatin sensitivity 
A

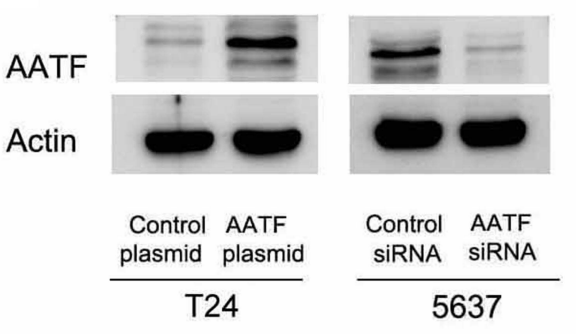

T24

B

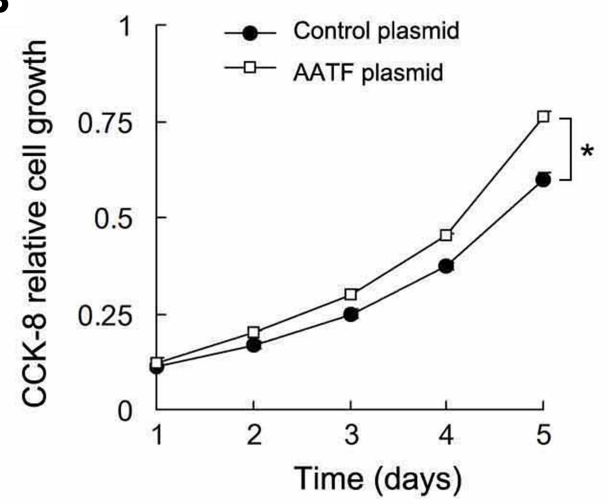

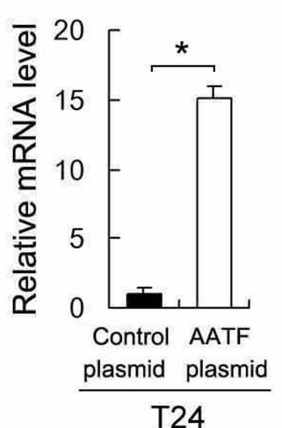

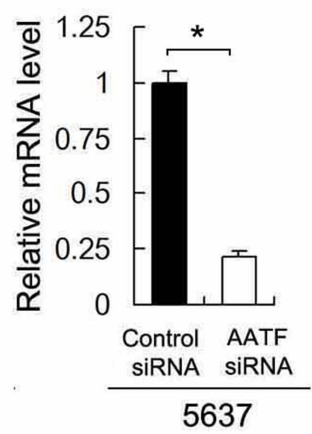

5637

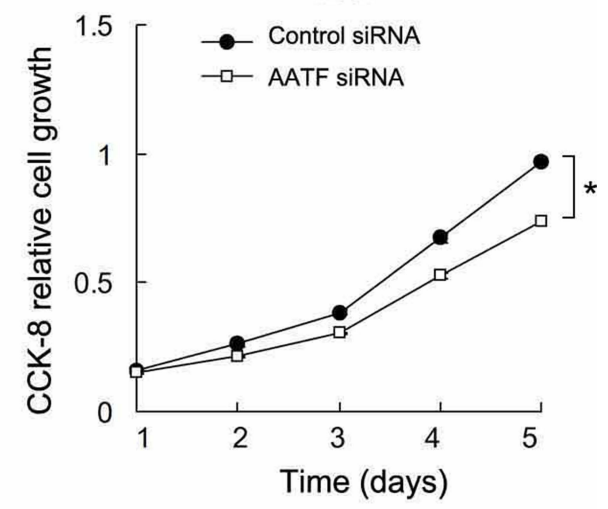

C

T24

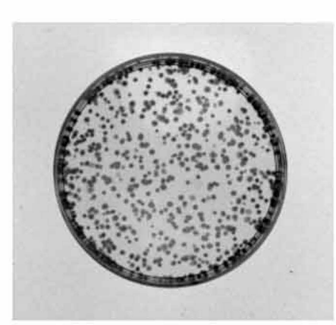

Control plasmid

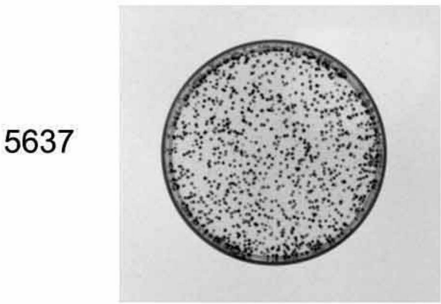

Control siRNA

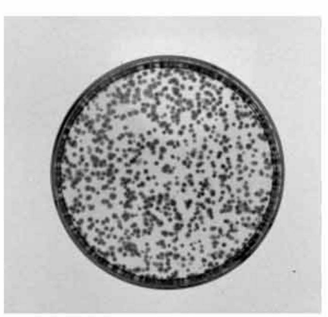

AATF plasmid

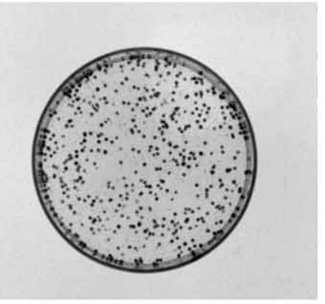

AATF siRNA
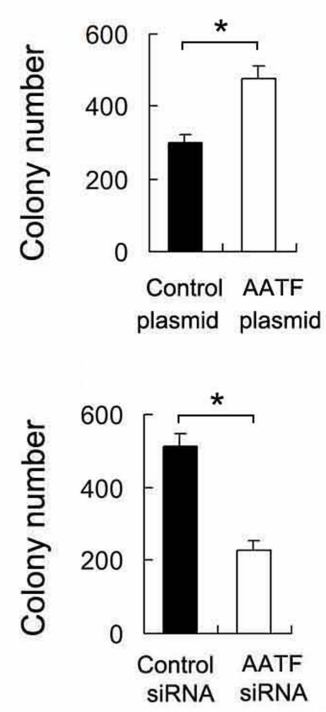

Figure 2 AATF positively regulates GC cell proliferation. (A) Transfection and siRNA knockdown efficiencies were confirmed by Western blot and RT-qPCR in T24 and 5637 cell lines, respectively. (B) CCK8 showed that growth rate was increased in T24 cells with AATF overexpression, while the cell growth rate was decreased in 5637 cells with AATF siRNA knockdown. (C) Colony formation assay demonstrated that colony counts were higher in T24 cells with AATF overexpression. AATF knockdown downregulated colony numbers formed by 5637 cell line. $* p<0.05$.

and decreased IC50 value compared with the control group (IC50 con vs AATF KD: $12.3 \mu \mathrm{M}$ vs $10.1 \mu \mathrm{M}$, p < 0.05) (Figure 3B). CCK-8 assay also showed that
AATF knockdown increased the cisplatin inhibition rate while AATF overexpression decreased it after 24 and $48 \mathrm{~h}$ of treatment (Figure 4A). Annexin V/PI 
A

T24

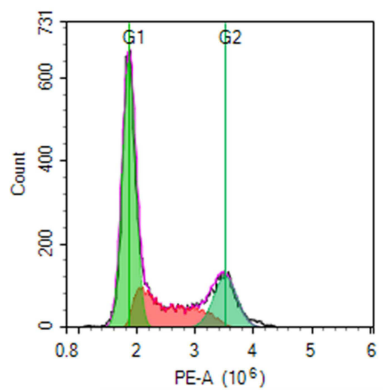

Control plasmid

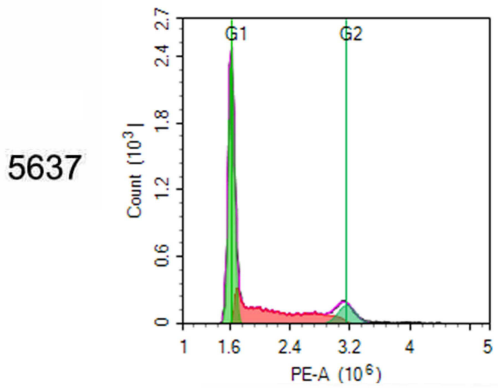

Control siRNA

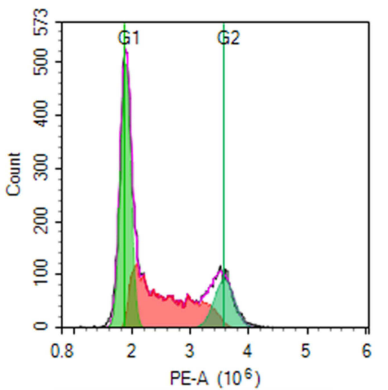

AATF plasmid

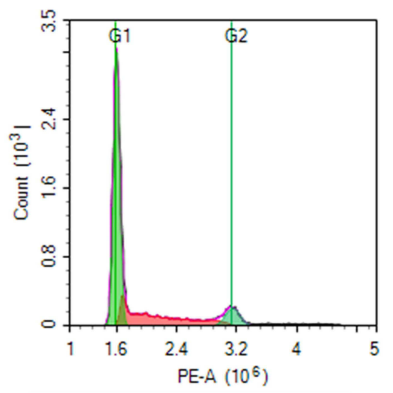

AATF SIRNA
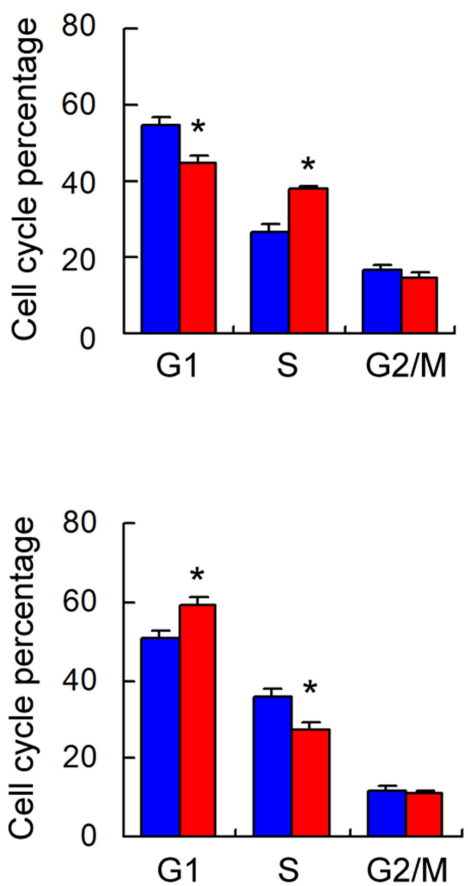

B
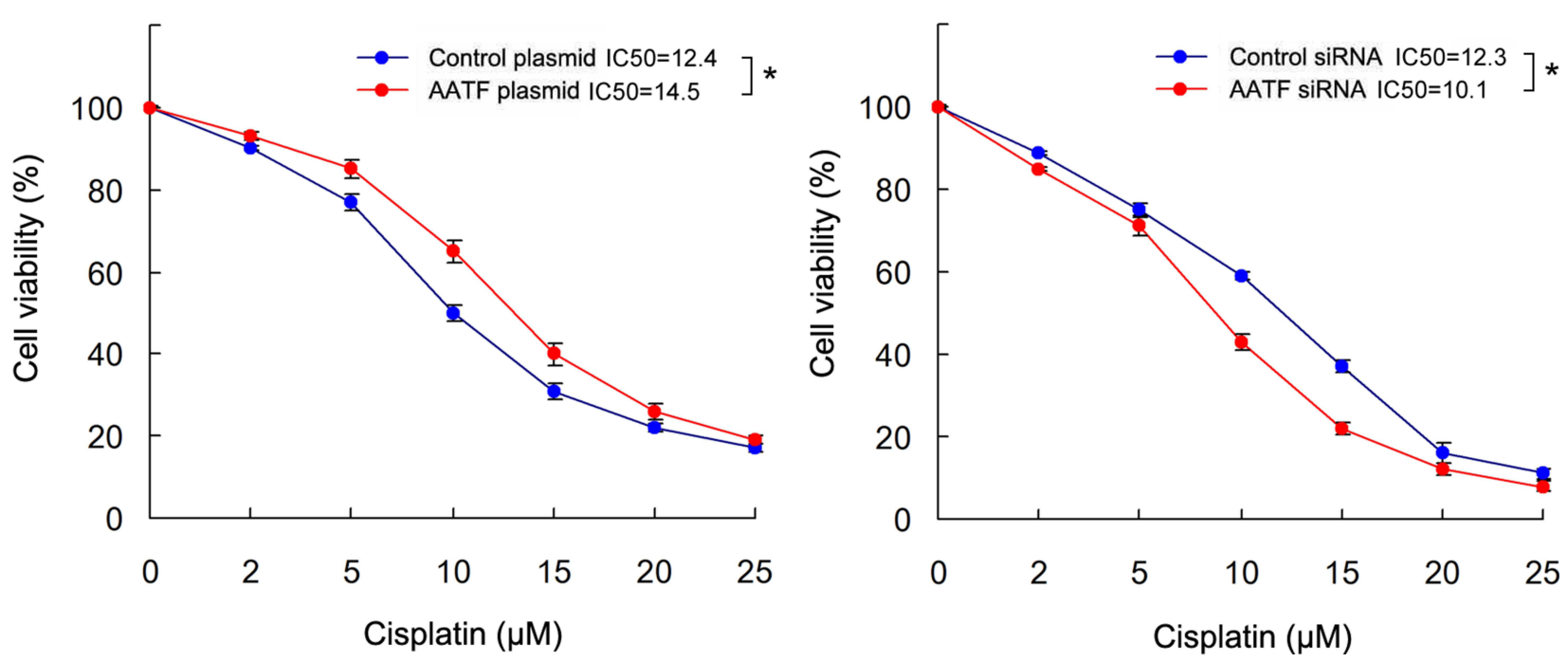

Figure 3 The effects of AATF on cell cycle and cisplatin inhibitory rate. (A) Cell cycle analysis showed that AATF overexpression upregulated S percentage in T24 cell line, while AATF siRNA decreased S percentage in 5637 cells. (B) The inhibitory rate of different cisplatin concentration in T24, T24/AATF, 5637, and 5637/AATFKD cells after

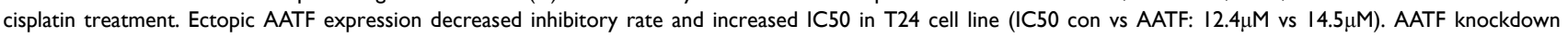

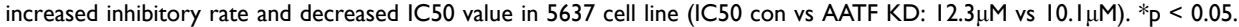

apoptosis assay indicated that AATF overexpression decreased the cisplatin-induced apoptosis $(10 \mu \mathrm{M}, 24 \mathrm{~h}$ cisplatin treatment) in T24 cell line. AATF depletion upregulated cisplatin-induced apoptosis $(10 \mu \mathrm{M}, 24 \mathrm{~h}$ cisplatin treatment) rate in 5637 cell line (Figure 4B).

\section{AATF Regulated Mitochondrial Membrane Potential and Cytochrome C}

Since mitochondrial function play an important role during cisplatin-induced apoptosis, we investigated whether AATF could regulate mitochondrial membrane potential. 

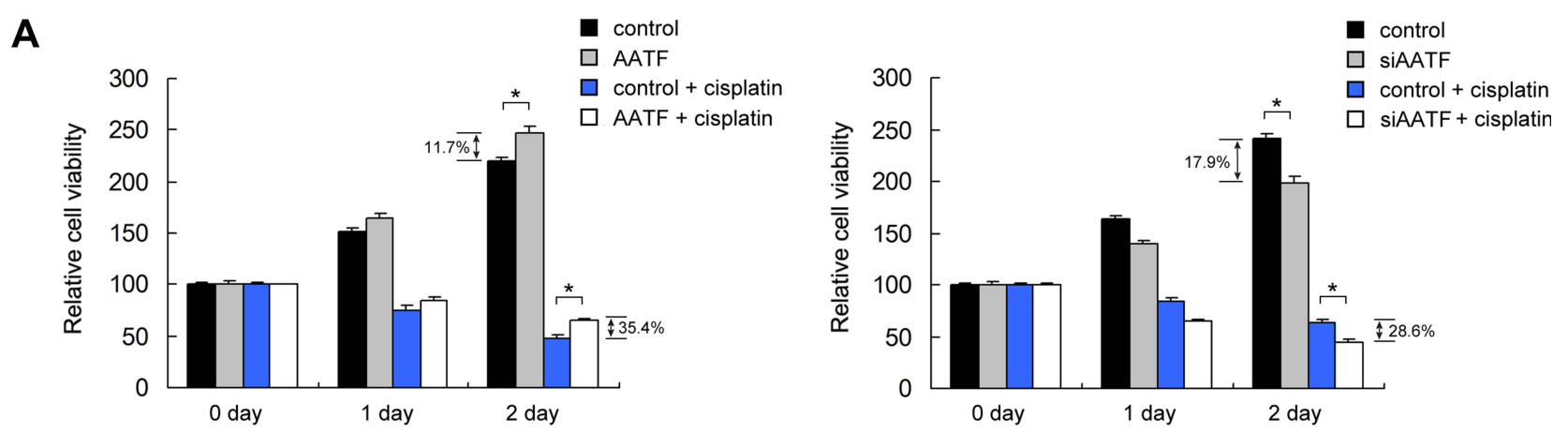

B
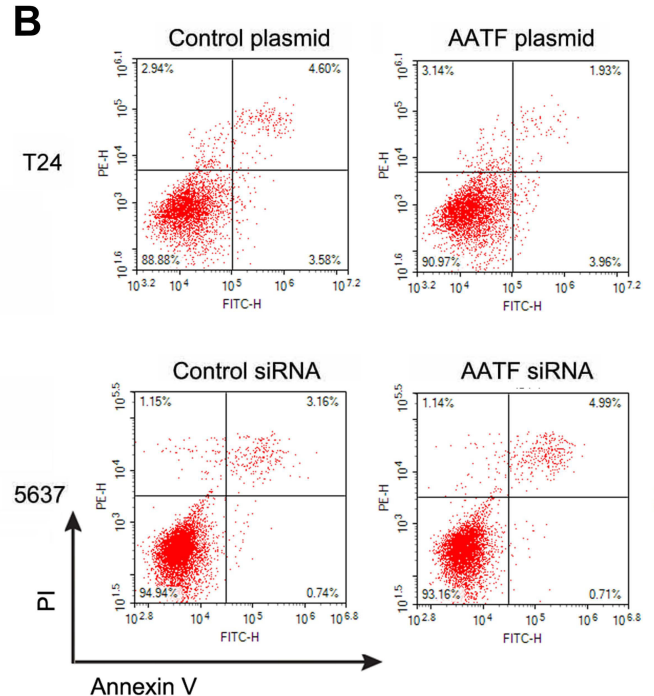

Annexin V

\section{C}
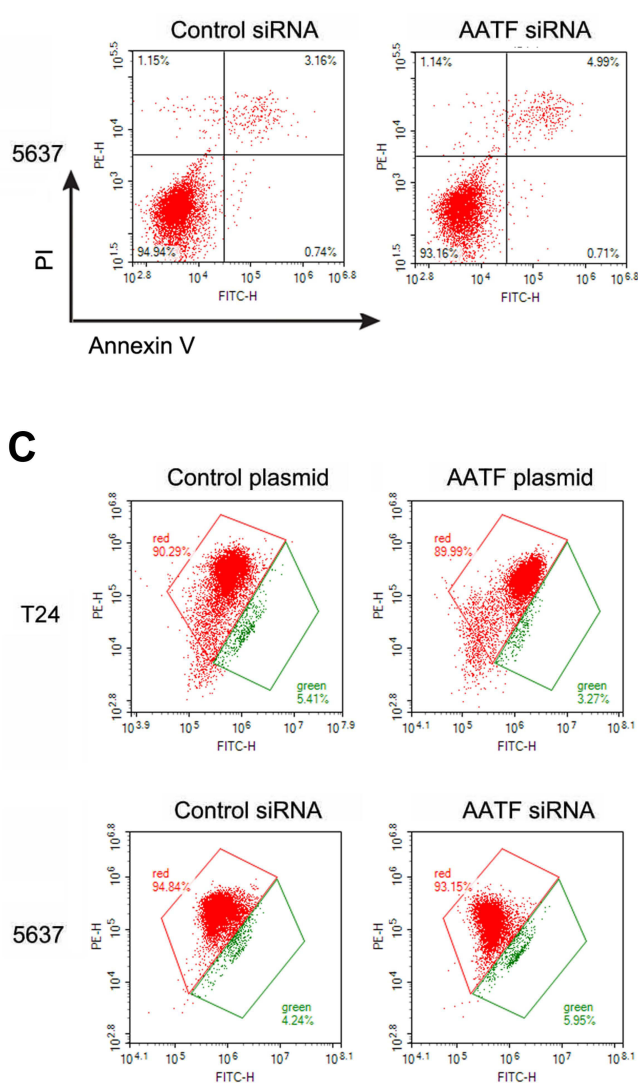
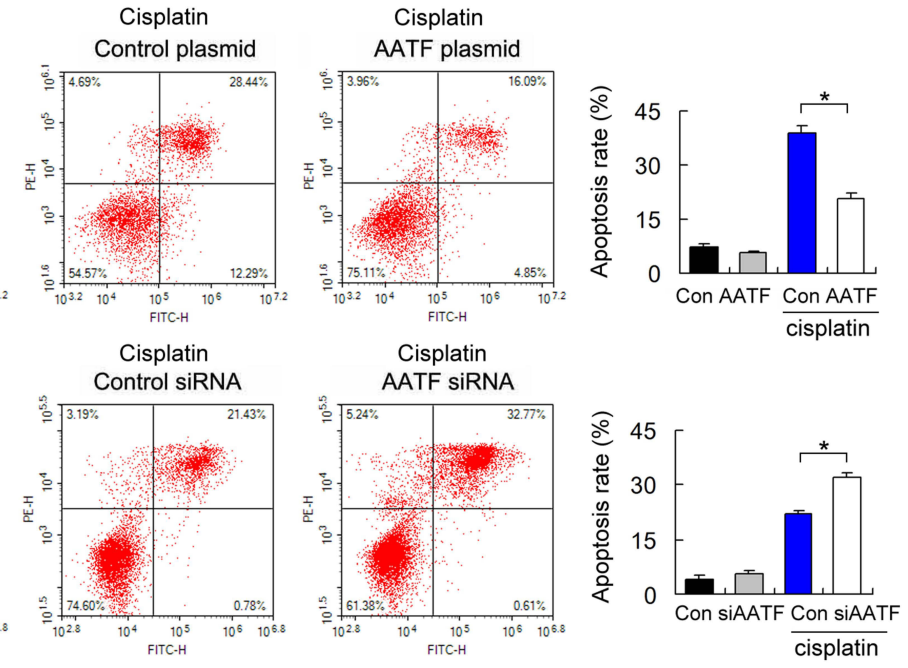

Figure 4 AATF regulates cisplatin sensitivity and apoptosis. (A) CCK8 cell viability assay showed that AATF overexpression increased cell viability while AATF knockdown decreased it after 24 and $48 \mathrm{~h}$ of cisplatin $(10 \mu \mathrm{M})$ treatment. The viability in cells without cisplatin was also shown. The fold changes of cell viability were indicated. (B) Annexin V/PI results showed that the cisplatin induced apoptosis rate was decreased in T24 cells transfected with AATF plasmid. AATF depletion upregulated cisplatin induced apoptosis in 5637 cell line. (C) JC-I staining showed that ectopic AATF expression upregulated the mitochondrial membrane potential by decreasing green fluorescence percentage in T24 cells treated with cisplatin. AATF knockdown downregulated the mitochondrial membrane potential by increasing green fluorescence in 5637 cells treated with cisplatin. The changes of membrane potential in non-treated cells were not significant. ${ }^{*} \mathrm{p}<0.05$.
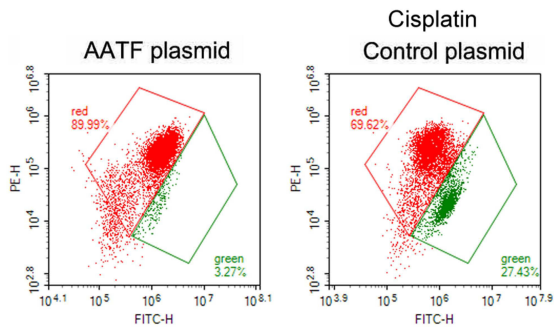

Cisplatin
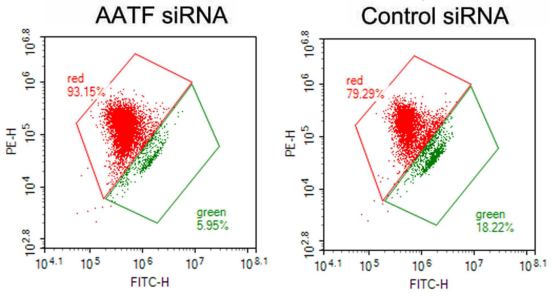
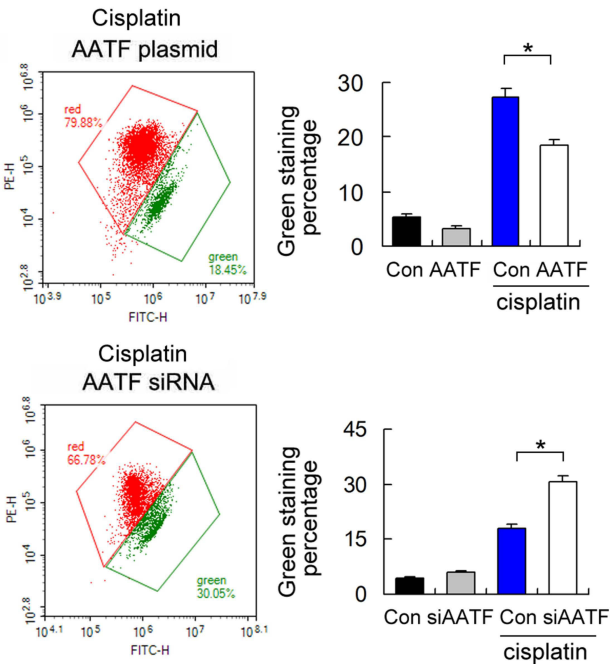
We used JC-1 staining with flow cytometry, which shows red fluorescence under normal condition and turns into green when mitochondrial membrane potential was impaired. As shown in Figure 4C, ectopic AATF expression downregulated the rate of green fluorescence, suggesting that AATF upregulated mitochondrial membrane potential in T24 cell line treated with cisplatin. On the other hand, AATF depletion downregulated mitochondrial membrane potential in cisplatin-treated 5637 cells. We also checked the levels of related proteins. AATF overexpression decreased the levels of cytochrome $\mathrm{c}$ and cleaved Poly(ADP-ribose) polymerase (PARP) in cisplatin treated T24 cell line. AATF depletion increased the levels of cytochrome $\mathrm{c}$ and cleaved PARP in cisplatin treated 5637 cell line (Figure 5A).

\section{AATF Regulated Cyclin E and Survivin in Bladder Cancer Cells}

To determine the possible mechanism of AATF in BC proliferation and chemo-sensitivity, we explored
A

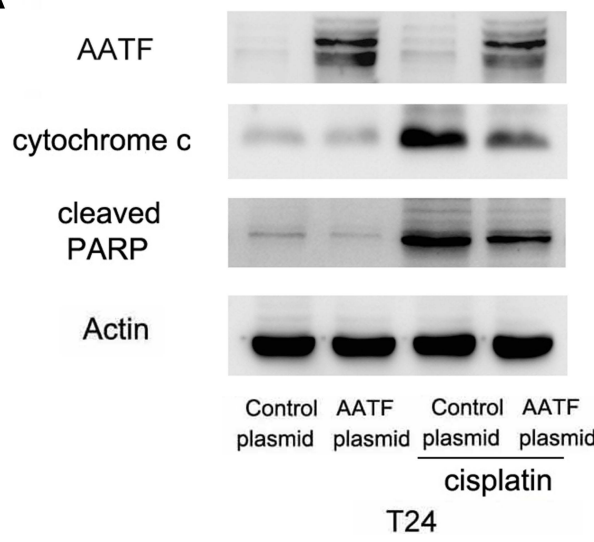

B

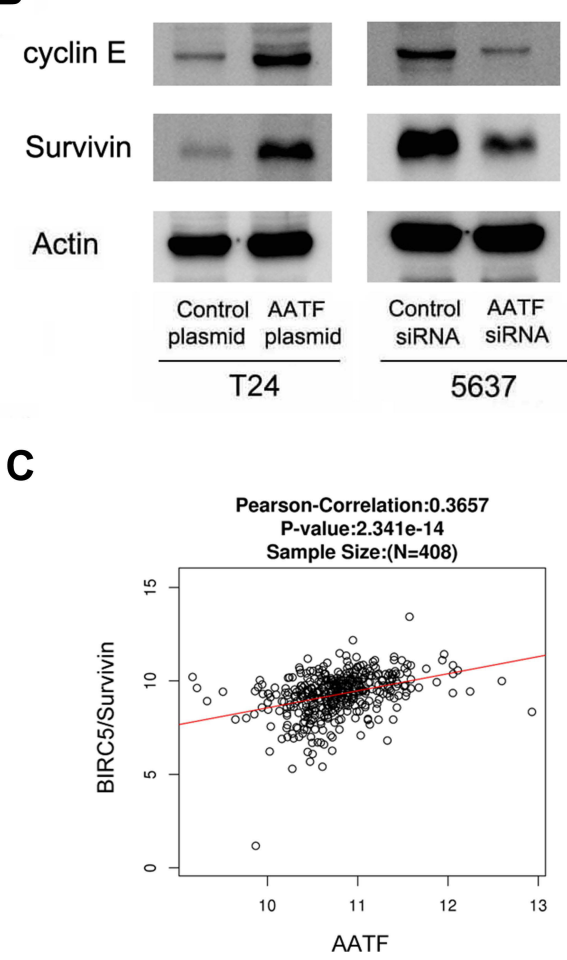

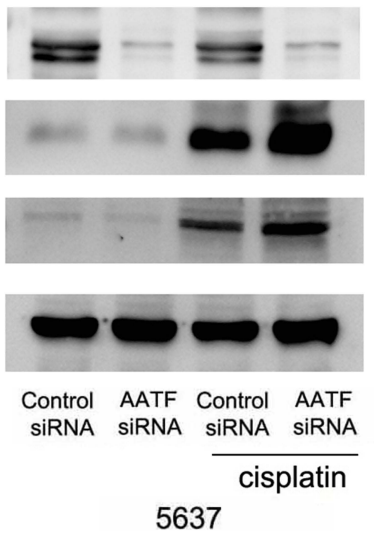
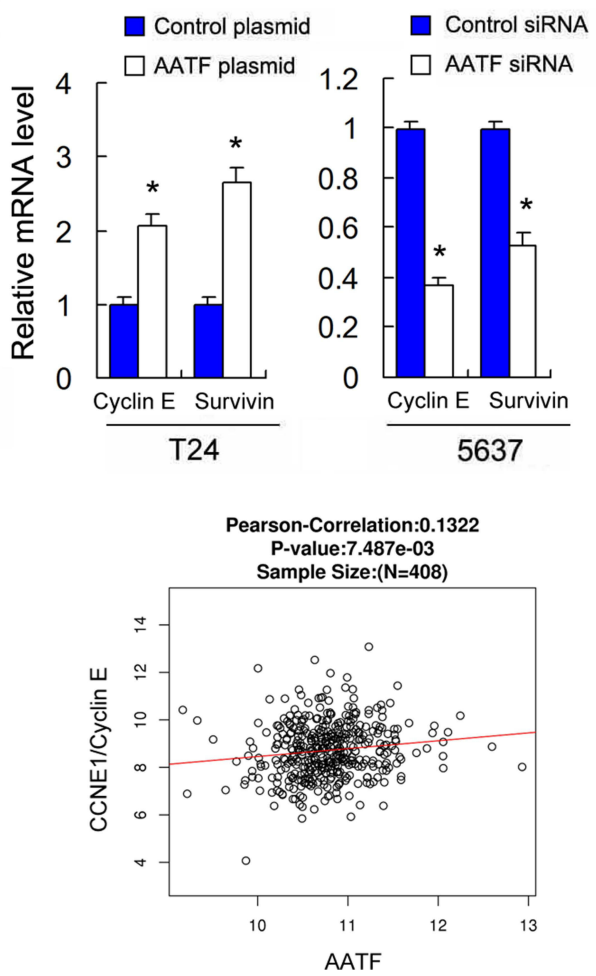

Figure 5 AATF regulates cyclin E and Survivin. (A) Western blotting showed that ectopic AATF expression decreased the levels of cytochrome c and cleaved PARP in cisplatin treated T24 cell line. AATF depletion increased the levels of cytochrome c and cleaved PARP in cisplatin treated 5637 cell line. (B) Western blotting and RT-qPCR showed ectopic AATF expression upregulated cyclin E and Survivin. AATF depletion downregulated cyclin E and Survivin at both mRNA and protein levels. (C) Analysis of TCGA data showed that AATF positively correlated with both cyclin E and Survivin mRNA in 408 cases of BC tissues. *p $<0.05$. 
potentially downstream proteins. Western blotting and RTqPCR showed that AATF overexpression upregulated cyclin E and Survivin protein and mRNA in T24 cells. On the contrary, AATF depletion downregulated cyclin $\mathrm{E}$ and Survivin in 5637 cells (Figure 5B). We also examined the correlations between AATF and Survivin/cyclin $\mathrm{E}$ in $\mathrm{BC}$ tissue samples by analyzing RNA-sequencing results of $408 \mathrm{BC}$ cases from TCGA. The result showed that there were statistically significant positive correlations between AATF with both Survivin and cyclin E (Pearson's correlation, Figure 5C), which further supported our results that AATF was a positive regulator of Survivin and cyclin E. In addition, BC cells were transfected with a survivin promoter firefly luciferase vector together with AATF plasmid or siRNA for $48 \mathrm{~h}$ to analyze changes of Survivin promoter activity. The results showed that AATF overexpression upregulated while AATF knockdown reduced Survivin promoter activity (Supplementary Figure 2D).

We also analyzed the clinical significance of Survivin bladder cancer using TCGA. As shown in Supplementary Figure 3A, Survivin/BIRC5 expression was significantly higher in $\mathrm{BC}$ tissues compared with normal tissues. Survival analysis showed that patients with high Survivin expression tends to have shorter overall survival compared with those with lower Survivin, although it did not reach a statistical significance (Log-rank, $\mathrm{p}=0.1155$, Supplementary Figure 3B). We also analyzed its relationship with clinical $\mathrm{T}$ stage (Supplementary Figure 3C), pathological M stage (Supplementary Figure 3D), pathological N stage (Supplementary Figure 3E), pathological $\mathrm{T}$ stage (Supplementary Figure 3F), pathological TNM stage (Supplementary Figure 3G) and histologic grade (Supplementary Figure 3H). Survival/BIRC5 did not showed significant correlation with these clinicopathological factors.

To further validate if Survivin was involved in cisplatin sensitivity regulated by AATF, we treated AATF transfected T24 cells with Survivin inhibitor YM155 (20 nM, $24 \mathrm{~h})$ and cisplatin $(10 \mu \mathrm{M})$. CCK-8 assay demonstrated that Survivin inhibitor reversed the effects of AATF on cisplatin sensitivity (Figure 6A). T24 cells treated with YM155 also showed decreased IC50 and AATF overexpression could not significantly increased IC50 in YM155 treated cells (Figure 6B).

Annexin V/PI analysis also demonstrated that Survivin inhibitor YM155 (20 nM, 24h) increased cisplatin-induced apoptosis $(10 \mu \mathrm{M}, 24 \mathrm{~h}$ treatment $)$ that was decreased by
AATF (Figure 6C). In T24 cells treated with YM155, the apoptosis reducing effect of AATF overexpression was abolished (Figure 6C). These results indicated that AATF regulated $\mathrm{BC}$ drug sensitivity through, at least partly, the regulation of Survivin.

\section{Discussion}

Recent studies have implicated AATF functions as a cancer-related protein. ${ }^{9}$ To date, the expression pattern of AATF in bladder cancers remains unexplored. In addition, its impact on biological behavior of $\mathrm{BC}$ cancer cells, such as chemo-sensitivity, has not been fully investigated. For the first time, our study showed the involvement of AATF in the regulation of proliferation and chemosensitivity in $\mathrm{BC}$ cells.

It has been recently reported AATF amplification in neuroblastoma was associated with poor patient prognosis. ${ }^{11}$ AATF expression is elevated in hepatocellular carcinoma and contributes to poor prognosis. ${ }^{15}$ Our results showed that AATF was overexpressed in $48.6 \%$ bladder cancer tissues which positively associated with $\mathrm{T}$ stage. This was also supported by data from Oncomine showing AATF upregulation in BC tissues. Importantly, TCGA data analysis showed that high levels of AATF associated with poor survival, making AATF a potential prognostic marker in $\mathrm{BC}$.

To explore the biological roles of AATF, we first screened normal uroepithelial and bladder cancer cell lines for its endogenous expression. AATF levels in cancer cell lines were higher compared with that in normal uroepithelial cell line. CCK-8 and colony formation assays demonstrated that AATF positively regulated BC cell proliferation. Indeed, AATF expression seems to interfere with cycling of T24 and 5637 cells. The S-phase increase in AATF plasmid-transfected T24 cells likely reveals an S-phase block during AATG gene overexpression, whereas the G1-phase increase in AATF siRNA oligo-transfected 5637 cells unveils a G1-phase block during AATF gene silencing. An obvious sign of proliferation increase due to changes in AATF expression is the change of cyclin E levels of expression. TCGA data analysis also revealed a positive association between AATF and cyclin E mRNA in $\mathrm{BC}$ tissues. Cyclin $\mathrm{E}$ plays a key role during regulation of the cell cycle. High expression of cyclin E shortens the G1 phase and promotes cancer cell proliferation. ${ }^{16}$ The above results suggested that AATF facilitated malignant proliferation of $\mathrm{BC}$ cells, possibly by positive regulation of cell cycle protein cyclin $\mathrm{E}$. 
A

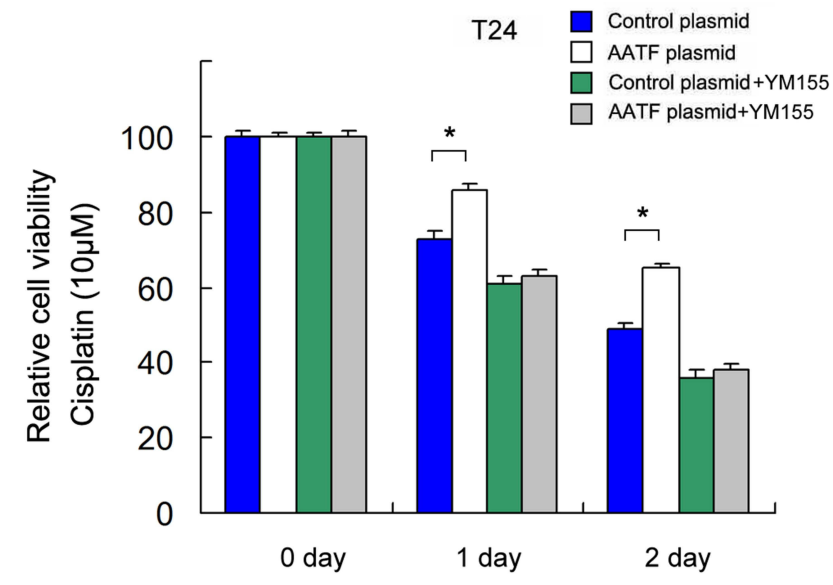

C

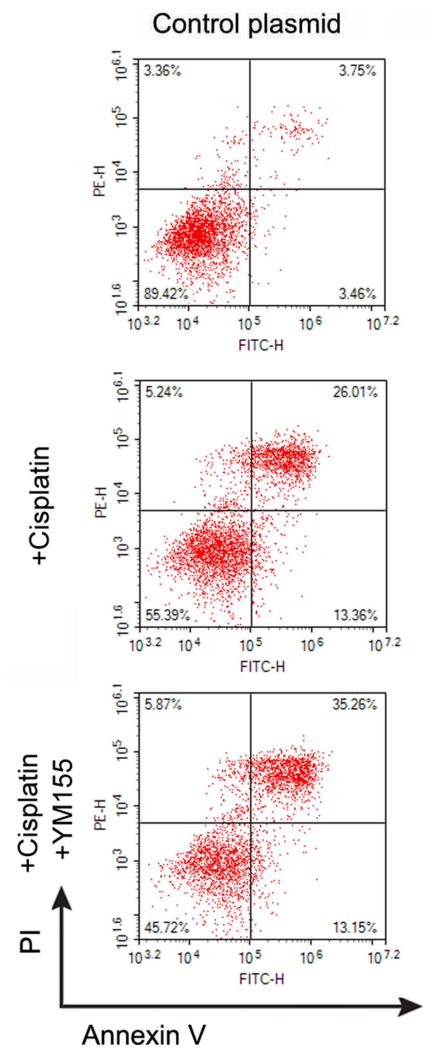

AATF plasmid
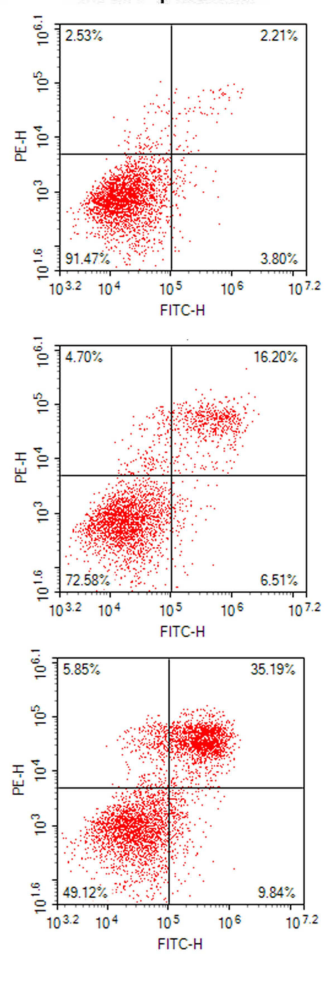

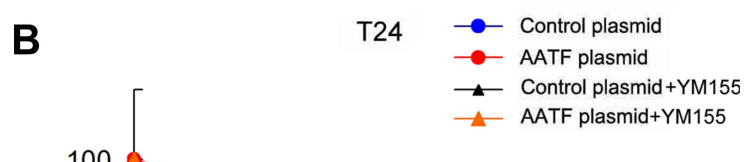

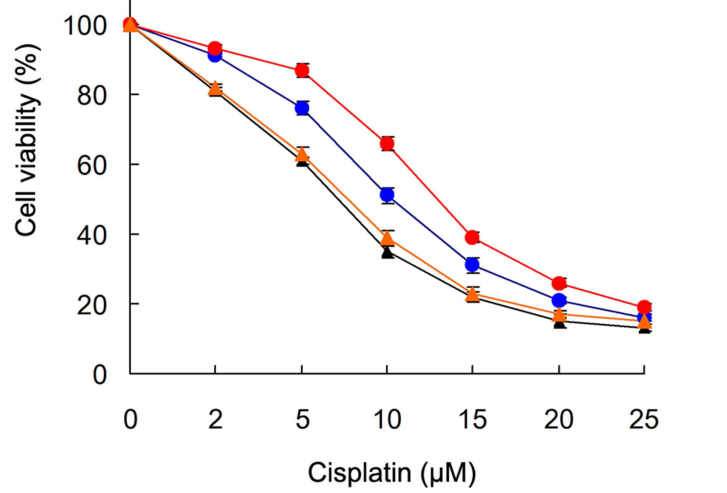

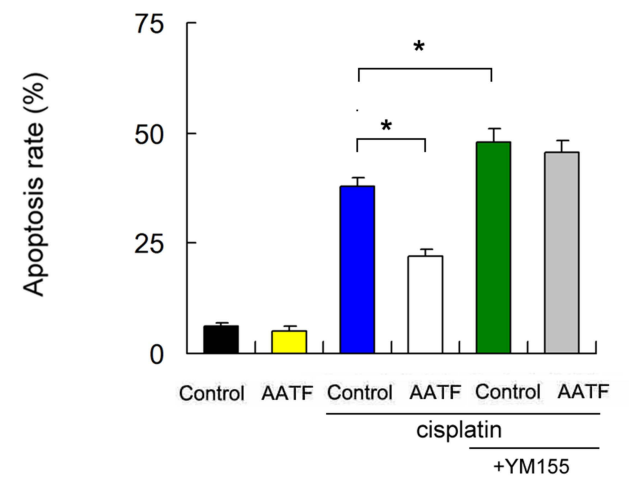

Figure 6 AATF regulates cisplatin-induced apoptosis through Survivin. (A) T24 cells with AATF overexpression were treated with Survivin inhibitor YMI 55 (I0 $\mu$ M) CCK-8 showed that AATF overexpression increased cell viability, which was abolished after treatment with YMI55. Survivin inhibitor reversed the effect of AATF on cisplatin sensitivity. (B) The inhibitory rate of different cisplatin concentration in T24, T24/AATF, T24/YMI55, and T24/AATF/YMI55 cells after cisplatin treatment. T24 cells treated with YMI55 also showed decreased IC50 and AATF overexpression could not significantly increased IC50 in YMI55 treated cells. (C) Apoptosis assay showed that Survivin inhibitor YMI55 upregulated cisplatin-induced apoptosis rate which was decreased by AATF. In T24 cells treated with YMI55, the apoptosis rate was not changed significantly after AATF overexpression. *p $<0.05$.

We further explored the role of AATF in BC chemosensitivity and its underlying mechanisms. We found that AATF overexpression downregulated cisplatin sensitivity and cisplatin-induced apoptosis. AATF depletion increased cisplatin sensitivity and apoptosis. Our data showed, for the first time, the involvement of AATF in chemosensitivity of bladder cancer cells. Mitochondrial membrane potential is essential for various functions including the production of ATP. Mitochondria membrane potential also plays a pivotal role in apoptosis induced by 
chemotherapeutic drugs. ${ }^{17-20}$ Using JC-1 staining, our results showed that AATF overexpression increased mitochondrial membrane potential in BC cells treated with cisplatin, suggesting AATF could stabilize mitochondrial function during apoptotic process induced by chemotherapeutic drugs.

Cytochrome $\mathrm{c}$ is a peripheral protein of the mitochondrial inner membrane and plays an essential role in generation of mitochondrial membrane potential. ${ }^{21}$ Loss of mitochondrial membrane potential and associated mitochondrial dysfunction could result in the release of cytochrome $\mathrm{c}$, which triggers cleavage of PARP. ${ }^{22}$ This is in accord with our results showing AATF reduced the levels of cytochrome $c$ and PARP cleavage while AATF depletion increased the levels of cytochrome $\mathrm{c}$ and PARP cleavage in cisplatin treated BC cells. The above data supported the roles of AATF to inhibit drug-induced apoptosis, possibly by maintaining mitochondrial membrane potential and reducing cytochrome c release.

We further explored the underlying mechanisms by which AATF regulates chemo-sensitivity. After screening a series of possible proteins, we showed that AATF overexpression upregulated Survivin protein and mRNA. Survivin/BIRC5 is a IAP family member protein which localizes in the mitochondria functions as an apoptosis inhibitor protein. ${ }^{23,24}$ Previous studies indicated that Survivin could maintain mitochondrial function and protect cancer cell from cell death induced by chemotherapeutic drugs. ${ }^{25}$ Survivin expression indicates worse prognosis in patients with bladder cancer. ${ }^{26}$ Survivin has also been reported to be crucial for the development of chemoresistance in bladder cancer. ${ }^{27}$ Our data further showed that decreased chemo-sensitivity and apoptosis induced by AATF were greatly impaired by Survivin inhibitor YM155. These results indicated that AATF could modulate cancer cell proliferation and cisplatin sensitivity through, at least partly, regulation of Survivin signaling.

A possible complication in this system may be the fact that the cell lines used in the study bear a mutant p53, while the AATF/Che-1 transcription factor has been proposed to act as a regulator of the cellular outcome of the p53 response, through binding on Puma, Bax and Bak promoter regions to repress p53-driven expression of the above pro-apoptotic genes. ${ }^{11}$ But, in p53-deficient cells such as T24 and 5637, upregulation of apoptosis must be of course p53-independent, which possibly suggests additional functions of the AATF.
In conclusion, this study identified AATF as a cancerrelated protein overexpressed in bladder cancer. AATF overexpression promoted proliferation and reduced cisplatin sensitivity in bladder cancers. We also linked its oncogenic effects with cyclin E and Survivin, suggesting the therapeutic possibility of targeting AATF in human bladder cancers.

\section{Acknowledgment}

The study was supported by The National Natural Science Foundation of China (No. 81772468) to Qianze Dong and The National Natural Science Foundation of China (No. 82103679) to Shutao Tan.

\section{Disclosure}

The authors report no conflicts of interest.

\section{References}

1. Siegel RL, Miller KD, Jemal A. Cancer statistics, 2018. CA Cancer J Clin. 2018;68:7-30.

2. Siegel RL, Miller KD, Jemal A. Cancer statistics, 2020. CA Cancer J Clin. 2020;70:7-30.

3. Fujita N, Hatakeyama S, Momota M, et al. Preoperative chronic kidney disease predicts poor prognosis in patients with primary non-muscle-invasive bladder cancer who underwent transurethral resection of bladder tumor. Urol Oncol. 2020;38:684e1- e8.

4. Yin M, Joshi M, Meijer RP, et al. Neoadjuvant chemotherapy for muscle-invasive bladder cancer: a systematic review and two-step meta-analysis. Oncologist. 2016;21:708-715.

5. Dong Q, Fu L, Zhao Y, Tan S, Wang E. Derlin-1 overexpression confers poor prognosis in muscle invasive bladder cancer and contributes to chemoresistance and invasion through PI3K/AKT and ERK/MMP signaling. Oncotarget. 2017;8:17059-17069.

6. Srinivas AN, Suresh D, Mirshahi F, Santhekadur PK, Sanyal AJ, Kumar DP. Emerging roles of AATF: checkpoint signaling and beyond. J Cell Physiol. 2021;236:3383-3395.

7. Bruno T, Iezzi S, De Nicola F, et al. Che-1 activates XIAP expression in response to DNA damage. Cell Death Differ. 2008;15:515-520.

8. Kumar DP, Santhekadur PK, Seneshaw M, et al. Role of apoptosis antagonizing transcription factor in the pathogenesis of nonalcoholic fatty liver disease and hepatocellular carcinoma. Hepatology. 2019;69:1520-1534.

9. Iezzi S, Fanciulli M. Discovering Che-1/AATF: a new attractive target for cancer therapy. Front Genet. 2015;6:141.

10. Bruno T, Iezzi S, Fanciulli M. Che-1/AATF: a critical cofactor for both wild-type- and Mutant-p53 proteins. Front Oncol. 2016;6:34.

11. Hopker K, Hagmann H, Khurshid S, et al. AATF/Che-1 acts as a phosphorylation-dependent molecular modulator to repress p53-driven apoptosis. EMBO J. 2012;31:3961-3975.

12. Bruno T, De Nicola F, Corleone G, et al. Che-1/AATF-induced transcriptionally active chromatin promotes cell proliferation in multiple myeloma. Blood Adv. 2020;4:5616-5630.

13. Remmele W, Stegner HE. [Recommendation for uniform definition of an immunoreactive score (IRS) for immunohistochemical estrogen receptor detection (ER-ICA) in breast cancer tissue]. Der Pathologe. 1987;8:138-140. German.

14. Fedchenko N, Reifenrath J. Different approaches for interpretation and reporting of immunohistochemistry analysis results in the bone tissue - a review. Diagn Pathol. 2014;9:221. 
15. Liu J, Lu J, Ma Z, Li W. A nomogram based on a three-gene signature derived from AATF coexpressed genes predicts overall survival of hepatocellular carcinoma patients. Biomed Res Int. 2020;2020:7310768.

16. Pang W, Li Y, Guo W, Shen H. Cyclin E: a potential treatment target to reverse cancer chemoresistance by regulating the cell cycle. $\mathrm{Am}$ J Transl Res. 2020;12:5170-5187.

17. Guerra F, Arbini AA, Moro L. Mitochondria and cancer chemoresistance. Biochim Biophys Acta. 2017;1858:686-699.

18. Kim JS, Lee JM, Chwae YJ, et al. Cisplatin-induced apoptosis in Hep3B cells: mitochondria-dependent and -independent pathways. Biochem Pharmacol. 2004;67:1459-1468.

19. Zhao W, You CC, Zhuang JP, et al. Viability inhibition effect of gambogic acid combined with cisplatin on osteosarcoma cells via mitochondria-independent apoptotic pathway. Mol Cell Biochem. 2013;382:243-252.

20. Li H, Fu L, Liu B, Lin X, Dong Q, Wang E. Ajuba overexpression regulates mitochondrial potential and glucose uptake through YAP/Bcl-xL/GLUT1 in human gastric cancer. Gene. 2019;693:16-24.

21. Ow YP, Green DR, Hao Z, Mak TW. Cytochrome c: functions beyond respiration. Nat Rev Mol Cell Biol. 2008;9:532-542.
22. Chen X, Wong JY, Wong P, Radany EH. Low-dose valproic acid enhances radiosensitivity of prostate cancer through acetylated p53-dependent modulation of mitochondrial membrane potential and apoptosis. Mol Cancer Res. 2011;9:448-461.

23. Waligorska-Stachura J, Jankowska A, Wasko R, et al. Survivinprognostic tumor biomarker in human neoplasms-review. Ginekol Pol. 2012;83:537-540.

24. Liu JL, Gao W, Kang QM, Zhang XJ, Yang SG. Prognostic value of survivin in patients with gastric cancer: a systematic review with meta-analysis. PLoS One. 2013;8:e71930.

25. Sattari M, Pazhang Y, Imani M. Calprotectin induces cell death in human prostate cancer cell (LNCaP) through survivin protein alteration. Cell Biol Int. 2014;38:1311-1320.

26. Akhtar M, Gallagher L, Rohan S. Survivin: role in diagnosis, prognosis, and treatment of bladder cancer. Adv Anat Pathol. 2006;13:122-126

27. Krafft U, Tschirdewahn S, Hess J, et al. Validation of survivin and HMGA2 as biomarkers for cisplatin resistance in bladder cancer. Urol Oncol. 2019;37:810e7- e15.

\section{Publish your work in this journal}

OncoTargets and Therapy is an international, peer-reviewed, open access journal focusing on the pathological basis of all cancers, potential targets for therapy and treatment protocols employed to improve the management of cancer patients. The journal also focuses on the impact of management programs and new therapeutic agents and protocols on patient perspectives such as quality of life, adherence and satisfaction. The manuscript management system is completely online and includes a very quick and fair peer-review system, which is all easy to use. Visit http://www.dovepress.com/ testimonials.php to read real quotes from published authors. 